\title{
Efficacy of Novel Ultrathin Single-Balloon Enteroscopy for Crohn's Disease: A Propensity Score-Matched Study
}

Kaoru Takabayashi ${ }^{1}$, Naoki Hosoe ${ }^{1}$, Motohiko Kato ${ }^{2,3}$, Yukie Hayashi ${ }^{1}$, Ryoichi Miyanaga ${ }^{1}$, Kosaku Nanki ${ }^{2}$, Kayoko

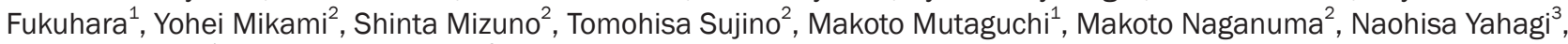
Haruhiko Ogata ${ }^{1}$, and Takanori Kanai ${ }^{2}$

${ }^{1}$ Center for Diagnostic and Therapeutic Endoscopy, ${ }^{2}$ Division of Gastroenterology and Hepatology, and ${ }^{3}$ Division of Research and Development for Minimally Invasive Treatment, Cancer Center, Department of Internal Medicine, Keio University School of Medicine, Tokyo, Japan

Background/Aims: The evaluation of small bowel lesions of Crohn's disease (CD) using balloon-assisted enteroscopy (BAE) is crucial because mucosal healing is associated with a good prognosis. However, BAE procedures are invasive, requiring sedation or analgesia to reduce the patient's pain. This study evaluated the clinical usefulness of a novel ultrathin single-balloon enteroscopy (SBE) procedure for CD. Methods: This single-center retrospective study included 102 CD patients who underwent trans-anal SBE between January 2012 and May 2018. Of these patients, 82 underwent enteroscopy using conventional SBE, while 20 underwent ultrathin SBE. Patients were analyzed using propensity score matching, with 20 patients per group. The median duration of the examination, terminal ileum intubation rate, median cecum intubation time, median insertion depth, adverse events, and sedated dose in each group were compared. Results: Before propensity score matching, the conventional SBE group had a larger number of surgical history patients than the ultrathin SBE group ( $p=0.05$ ). After matching, the two groups did not significantly differ clinically. There were no significant differences in the mean duration of the examination, cecum intubation time, or terminal ileal intubation rate between ultrathin SBE and conventional SBE. The mean insertion depth of ultrathin SBE tended to be deeper than that of conventional SBE ( $p=0.09)$. The use of ultrathin SBE also reduced the sedative dose during needed for enteroscopy compared with conventional SBE $(p=0.005)$. Conclusions: Novel ultrathin SBE may be less painful for CD patients than conventional SBE. (Gut Liver 2020;14:619-625)

Key Words: Enteroscopy; Single-balloon enteroscopy; Ultrathin scope; Crohn disease

\section{INTRODUCTION}

Crohn's disease (CD) is a chronic inflammatory disease that can involve the entire gastrointestinal tract. Small bowel lesions are observed in $40 \%$ to $60 \%$ of patients with $\mathrm{CD}$, but clinical symptoms do not predict the presence and severity of these active lesions. ${ }^{1-3}$ The assessment of active mucosal lesions in the small bowel is crucial in CD because mucosal healing is associated with a better prognosis, such as a lower rate of relapse, as well as decreased rates of hospitalization and surgical resection. $^{4-8}$

Several diagnostic methods are available to evaluate small bowel lesions in CD patients. These include esophagogastroduodenoscopy, ileocolonoscopy, video capsule endoscopy, balloonassisted enteroscopy (BAE), X-ray studies, high-resolution ultrasound, and cross-sectional radiologic imaging. ${ }^{9}$ Among these, BAE, including single-balloon enteroscopy (SBE) and doubleballoon enteroscopy, is recommended for evaluation of small bowel lesions because it not only allows direct visualization of the small bowel mucosa, but also allows biopsies to be obtained for histopathologic analysis. However, a disadvantage is that BAE procedures are more invasive than esophagogastroduodenoscopy, ileocolonoscopy, and video capsule endoscopy. BAE procedures usually require sedation or analgesia, and patients who undergo BAE, therefore, occasionally need to be hospitalized to recover from deep sedation. Furthermore, patients with $\mathrm{CD}$ often have a surgical history, and it is not unusual to have stenotic lesions in the gastrointestinal tract due to their disease activity. Therefore, the use of rigid sliding tubes can cause pain during the procedure, leading to reduced patient acceptance, especially under conscious sedation. Because of its invasiveness, BAE is not currently a gold standard tool for evaluating small

Correspondence to: Naoki Hosoe

Center for Diagnostic and Therapeutic Endoscopy, Keio University School of Medicine, 35 Shinanomachi, Shinjuku-ku, Tokyo 160-8582, Japan

Tel: +81-3353-3536, Fax: +81-3353-3536, E-mail: nhosoe@z5.keio.jp

Received on July 4, 2019. Revised on September 10, 2019. Accepted on October 1, 2019. Published online December $12,2019$.

pISSN 1976-2283 eISSN 2005-1212 https://doi.org/10.5009/gnl19228

(c) This is an Open Access article distributed under the terms of the Creative Commons Attribution Non-Commercial License (http://creativecommons.org/licenses/by-nc/4.0) which permits unrestricted non-commercial use, distribution, and reproduction in any medium, provided the original work is properly cited. 
bowel lesions in $\mathrm{CD} .^{10}$

The clinical utility of a thin endoscope for esophagogastroduodenoscopy and colonoscopy has been demonstrated in numerous studies. ${ }^{11-13}$ The main advantage of using a thin endoscope is its high comfortability and tolerability to the patient. Previously, we reported the clinical utility of novel ultrathin SBE as a feasibility modality. ${ }^{14}$ In this previous report, various types of patients were inspected using novel ultrathin SBEs; we ultimately concluded that novel ultrathin SBE provides adequate insertability and safety for surveillance enteroscopy under conscious sedation. Moreover, using novel ultrathin SBE has the potential benefit of decreasing the amount of sedative drug needed. As a result, novel ultrathin SBE may reduce patient discomfort during the procedure. From these results, we hypothesized that it would be possible to reduce patients' discomfort by using nove ultrathin SBE for patients with $\mathrm{CD}$. The present study aimed to evaluate the clinical usefulness of novel ultrathin SBE in CD patients by using propensity score-matched analysis.

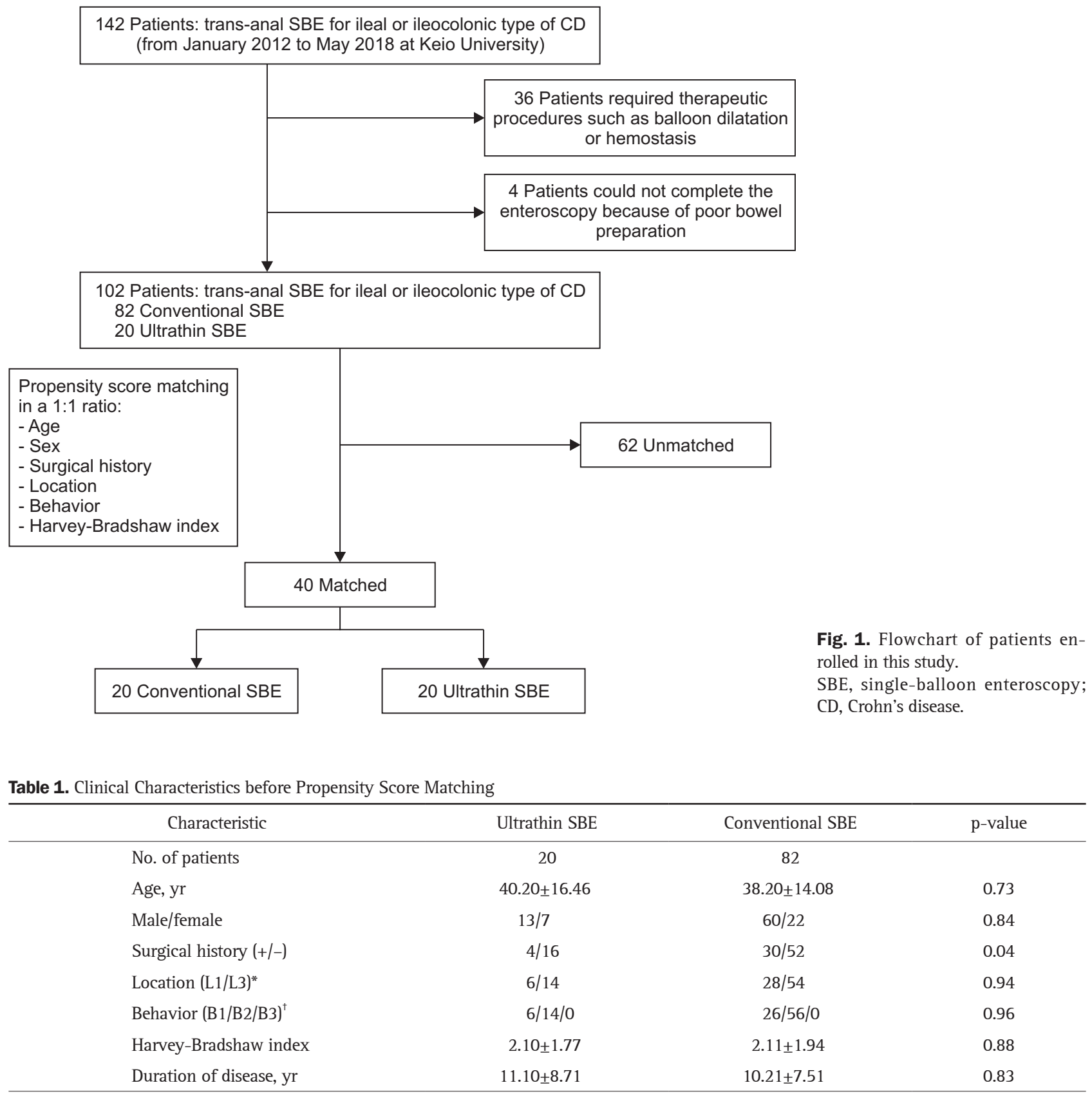

Data are presented as number or mean \pm SD. Surgical history: either ileocecal resection or partial resection of the small bowel.

SBE, single-balloon enteroscopy.

*Location L1, ileal; L3, ileocolonic; ${ }^{\dagger}$ Behavior B1, nonstricturing, nonpenetrating; B2, stricturing; B3, penetrating. 


\section{MATERIALS AND METHODS}

\section{Patients}

This was a propensity score-matched retrospective study. SBE has been indicated to CD patients who have been noted for possible small bowel lesions in other modalities, or who have previously had small bowel lesions. Of 142 consecutive ileal or ileocolonic type of $\mathrm{CD}$ patients who underwent trans-anal enteroscopy to evaluate small bowel lesion between Jan 2012 and May 2018 at Keio University Hospital, 102 CD patients were included in this study. Exclusion criteria were patients who required therapeutic procedures, such as balloon dilatation and hemostasis, and patients who could not complete the enteroscopy because of poor bowel preparation. The patients were divided into two groups: those who underwent conventional enteroscopy (SIF-Q260; Olympus, Tokyo, Japan) and those who underwent ultrathin enteroscopy (SIF-Y0018; Olympus). Propensity score matching was calculated for each group with bivariate logistic regression analysis by age, sex, surgical history, disease location, behavior, Harvey-Bradshaw index, and duration of disease. These propensity scores were used to match patients in using a conventional enteroscopy group in a 1:1 ratio with patients in using the ultrathin enteroscopy group (Fig. 1). The characteristics of the patients were compared between groups before and after the propensity score matching (Tables 1 and 2).

\section{Single-balloon enteroscopy}

The SBE system included a video enteroscope (SIF-Y0018 and SIF-Q260; Olympus), a sliding tube with a balloon (ST-Y0005 and ST-SB1; Olympus), and a balloon controller unit (Olympus). The features of the ultrathin SBE are compared with a conventional SBE in Table 3 and Fig. 2. The ultrathin enteroscope has a working length of 2,000 $\mathrm{mm}$, with a distal-end outer diameter of $5.4 \mathrm{~mm}$, insertion-tube outer diameter of $6.5 \mathrm{~mm}$, and a working channel diameter of $2.2 \mathrm{~mm}$, which only allowed the use of biopsy forceps. It has additional features including "passive bending," in which a secondary bending section, located close to the primary bending section at the distal end of the enteroscope, bends passively and is highly flexible. The features of the sliding tube are also shown in Table 3. The sliding tube for the ultrathin enteroscope had a working length of 1,320 mm with an outer diameter of $9.9 \mathrm{~mm}$ and an inner diameter of 7.7 $\mathrm{mm}$, but its balloon length and outer diameter were the same as the conventional sliding tube.

\section{Procedure}

Patients undergoing trans-anal enteroscopy received bowel preparation at least 4 hours before the procedure using a

Table 3. Features of Ultrathin Endoscopy/Sliding Tube and Conventional Enteroscopy/Sliding Tube

\begin{tabular}{|c|c|c|}
\hline Feature & Ultrathin & Conventional \\
\hline \multicolumn{3}{|l|}{ Enteroscopy } \\
\hline Observation range, $\mathrm{mm}$ & $3-100$ & $3-100$ \\
\hline Angle of view, ${ }^{\circ}$ & 140 & 140 \\
\hline Distal end outer diameter, $\mathrm{mm}$ & 5.4 & 9.2 \\
\hline Insertion tube outer diameter, $\mathrm{mm}$ & 6.5 & 9.2 \\
\hline \multicolumn{3}{|l|}{ Angle range, ${ }^{\circ}$} \\
\hline Up/down & $180 / 180$ & $180 / 180$ \\
\hline Right/left & $160 / 160$ & $160 / 160$ \\
\hline Channel inner diameter, mm & 2.2 & 2.8 \\
\hline Working length, mm & 2,000 & 2,000 \\
\hline Passive bending & + & - \\
\hline \multicolumn{3}{|l|}{ Sliding tube } \\
\hline Outer diameter, $\mathrm{mm}$ & 9.9 & 13.2 \\
\hline Inner diameter, mm & 7.7 & 11 \\
\hline Balloon outer diameter, $\mathrm{mm}$ & 40 & 40 \\
\hline Balloon length, mm & 52 & 52 \\
\hline Working length, mm & 1,320 & 1,320 \\
\hline Total length, mm & 1,400 & 1,400 \\
\hline Tube material & Silicone & Silicone \\
\hline Balloon material & Silicone & Silicone \\
\hline
\end{tabular}

Table 2. Clinical Characteristics after Propensity Score Matching

\begin{tabular}{lccc}
\hline \multicolumn{1}{c}{ Characteristic } & Ultrathin SBE & Conventional SBE & p-value \\
\hline No. of patients & 20 & 20 & 0.82 \\
Age, yr & $40.20 \pm 16.46$ & $38.90 \pm 17.79$ & 1.00 \\
Male/female & $13 / 7$ & $13 / 7$ & 1.00 \\
Surgical history (+/-) & $4 / 16$ & $4 / 16$ & 0.80 \\
Location (L1/L3) & $6 / 14$ & $7 / 13$ & 0.80 \\
Behavior (B1/B2/B3) ${ }^{\dagger}$ & $6 / 14 / 0$ & $7 / 13 / 0$ & 0.84 \\
Harvey-Bradshaw index & $2.10 \pm 1.77$ & $2.15 \pm 2.12$ & 0.88 \\
Duration of disease, yr & $11.10 \pm 8.71$ & $10.60 \pm 7.90$ & 0 \\
\hline
\end{tabular}

Data are presented as number or mean \pm SD. Surgical history: either ileocecal resection or partial resection of the small bowel.

SBE, single-balloon enteroscopy.

*Location L1, ileal; L3, ileocolonic; ${ }^{\dagger}$ Behavior B1, nonstricturing, nonpenetrating; B2, stricturing; B3, penetrating. 

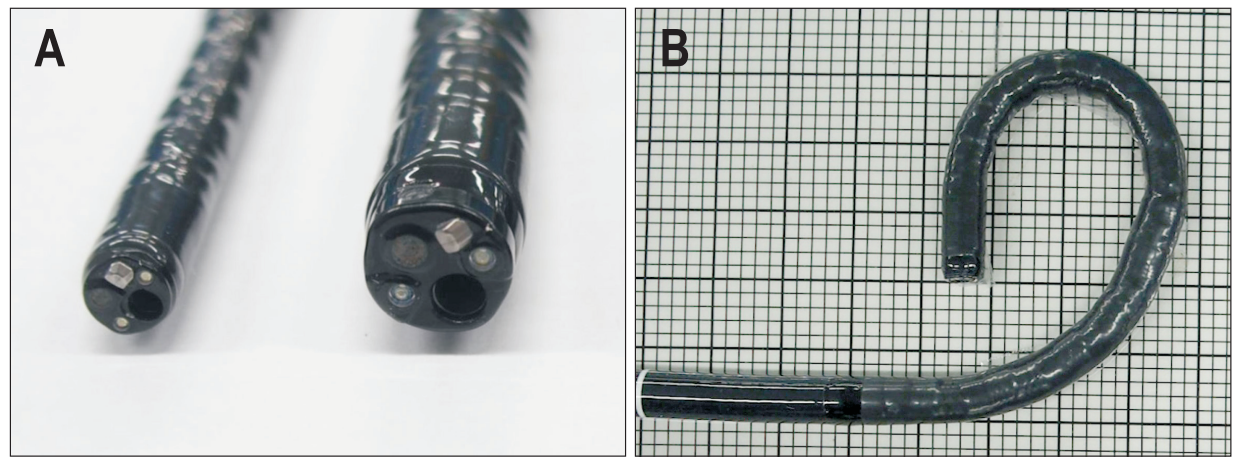

Fig. 2. (A) Ultrathin single-balloon enteroscopy (left) and conventional single-balloon enteroscopy (right). (B) Ultrathin single-balloon enteroscopy has additional features, including "passive bending," in which a secondary bending section is located close to the primary bending section at the distal end of the enteroscope.

Table 4. Technical Aspects of Ultrathin SBE and Conventional SBE

\begin{tabular}{lccc}
\hline \multicolumn{1}{c}{ Technical aspect } & Ultrathin SBE & Conventional SBE & p-value \\
\hline Duration of examination, min & $44.50 \pm 8.14$ & $41.20 \pm 13.10$ & 0.38 \\
Terminal ileum intubation rate, $\%$ & 100 & 95.0 & 0.58 \\
Cecal intubation time, min & $10.80 \pm 3.16$ & $13.50 \pm 6.09$ & 0.21 \\
Insertion depth beyond ICV or anastomosis, cm & $62.75 \pm 46.78$ & $46.5 \pm 48.67$ & None \\
Adverse events & None & 35.0 \\
Sedation & & 35.0 & 0.2 \\
Initial dose of pethidine hydrochloride, $\mathrm{mg}$ & 0.2 & $0.10 \pm 0.12$ & 0.005 \\
Initial dose of flunitrazepam, mg & $0.01 \pm 0.02$ &
\end{tabular}

Data are presented as mean \pm SD.

SBE, single-balloon enteroscopy; ICV, ileocecal valve.

polyethylene glycol lavage solution (Niflec or MOVIPREP; EA Pharma Co., Tokyo, Japan) after overnight fasting. All patients were consciously sedated with pethidine hydrochloride $35 \mathrm{mg}$ and flunitrazepam $0.2 \mathrm{mg}$, with intravenous scopolamine butylbromide (20 mg) or glucagon (1 $\mathrm{mg}$ ) as an antispasmodic given before the procedure to prevent digestive tract spasm. Cardiopulmonary function was monitored during the procedure. In cases of severe pain during the procedure, the operator added flunitrazepam in $0.2 \mathrm{mg}$ increments as required. All endoscopic examinations were carried out by five expert endoscopists who were Board Certified Fellows of the Japan Gastroenterological Endoscopy Society, and had at least more than 100 SBE experiences. All procedures were carried out by either the single- or dual-operator method. ${ }^{15}$ Information on patients' demographics, clinical data, procedural data, and adverse events was collected from medical charts, and details of the endoscopy procedures were collected using the endoscopy reporting system (Solemio ENDO $^{\circledR}$, Olympus). Total procedure time was defined as the time from insertion to the withdrawal of the enteroscope. Insertion depth is defined as the distance to the site where insertion to the deep part becomes difficult due to adhesion or stenosis of the intestinal tract, or the distance that can be inserted in 30 minutes. Insertion depth was measured in centimeters according to the method defined by May et al., ${ }^{16}$ or by measuring the amount of small bowel traversed on withdrawal in 5- or 10-cm increments. ${ }^{17}$ The study was carried out in accordance with approval of the ethics committee of Keio University (approval number: 20160431).

\section{Outcome measures}

The primary study outcome measure was the total sedative drug dose requirement during enteroscopy. Secondary study outcome measures were the mean duration of examination, terminal ileum intubation rate, mean cecum intubation time, mean insertion depth, and adverse events.

\section{Statistical analysis}

Statistical analysis was performed using JMP software version 11.1.1 (SAS Institute Inc., Cary, NC, USA). Results are summarized as medians and ranges for continuous variables, and categorical variables are summarized as numbers. The median values were compared between groups using the Mann-Whitney test or the chi-square test in univariate analysis. Statistical significance was defined as $\mathrm{p}<0.05$. 


\section{RESULTS}

\section{Characteristics before and after propensity score match- ing}

Before propensity score matching, the conventional SBE group had a larger number of patients with surgical history than the ultrathin SBE group ( $\mathrm{p}=0.04)$. After matching, the two groups included 20 patients, and did not significantly differ in any patient characteristics (Tables 1 and 2).

\section{Procedural outcomes}

Procedural outcomes are summarized in Table 4. There were no adverse events in either group. There were no significant differences in the mean duration of examination and mean cecal intubation time between two groups. Terminal ileum intubation rate was 95\% in the conventional SBE group compared with 100\% in the ultrathin SBE group. Of the 20 patients using conventional SBE, two patients had severe stenosis, which was difficult for both the sliding tube and the enteroscope to pass through at the ileocecal valve or anastomosis. In contrast, of the 20 patients examined using the ultrathin SBE, four patients had severe stenosis, which made it difficult for the ultrathin sliding tube to pass through at the ileocecal valve or anastomosis. However, the ultrathin enteroscope could pass through all of these stenoses, and could insert $30 \mathrm{~cm}$ deeper beyond the stenosis. The mean insertion depth of the ultrathin SBE tended to be deeper than that of the conventional SBE $(p=0.09)$. All patients were consciously sedated with pethidine hydrochloride $35 \mathrm{mg}$ and flunitrazepam $0.2 \mathrm{mg}$, and the mean dose of additional flunitrazepam was significantly lower in the ultrathin SBE compared with the conventional SBE group $(\mathrm{p}<0.05)$.

\section{DISCUSSION}

The clinical characteristics of CD include a long-term progressive course of repeated recurrence and remission of chronic inflammation. Based on previous reports, small bowel lesions are present in more than half of CD patients. ${ }^{1-3}$ The main pathological feature of early stage CD is gastrointestinal inflammation, but intestinal complications, such as stenosis, fistula, and abscess, develop with recurrences. Ultimately, surgery is indicated in many cases. Moreover, recurrent lesions from the anastomotic site occur at high rates even after intestinal resection, and some patients develop short bowel syndrome due to repeating intestinal resection. Therefore, it is crucial to accurately evaluate small bowel lesions and perform therapeutic intervention aiming at mucosal healing in the early stages. ${ }^{18}$

Recent European evidence-based consensus (European Crohn's and Colitis Organisation) guidelines recommend a capsule endoscopy or combination of ileocolonoscopy and crosssectional radiologic imaging represented by magnetic resonance enterography for evaluation of small bowel lesions in CD.
However, these tools have not been regarded as the gold standard evaluation method. Capsule endoscopy is a less invasive procedure and was reported a sensitive tool to detect mucosal abnormalities in the small bowel. For diagnosing small bowel $\mathrm{CD}$, the diagnostic yield of capsule endoscopy is thought to be more superior than other modalities. However, capsule endoscopy has a problem that it cannot be used for patients with a history of small bowel resection or known stenosis or patients with obstructive symptoms because of the risk of capsule retention. ${ }^{19}$ On the other hand, ileocolonoscopy can evaluate small bowel mucosa only up to about $10 \mathrm{~cm}$ proximal to the ileocecal valve. This insufficiency is partially overcome by combining this modality with magnetic resonance enterography. ${ }^{19}$ However, as previously reported, not all hospitals perform this as a standard examination because cooperation with the radiology department is essential for magnetic resonance enterography. In addition, a low detection rate of stenotic lesions has been noted, although the sensitivity and specificity for detecting ulcerative lesions are high. ${ }^{20-22}$ Therefore, in cases for which combining these two modalities is insufficient, guidelines advocate for evaluation of the small bowel using balloon endoscopy, which is capable of both direct observation and sampling for histological evaluation of lesions. ${ }^{19}$ However, double-balloon enteroscopy and SBE are more invasive than conventional endoscopy because the scope is inserted into the deep small bowel by concomitantly using a sliding tube with a large diameter; a minimally-invasive enteroscope has not yet been invented. ${ }^{10}$

In this study, we performed enteroscopy in CD patients using a novel ultrathin enteroscope and ultrathin sliding tube and compared the insertability, safety, and invasiveness with those of conventional SBE. In several previous studies using thin endoscopes in the upper and lower gastrointestinal tract, the maneuverability of the thin endoscope was poor, and its insertability was inferior to that of the conventional endoscope. ${ }^{23-25}$ The results of these reports suggested that deep insertion using the ultrathin enteroscope might extend the procedure time. To evaluate the efficacy and performance of ultrathin SBE, especially in CD patients, a propensity score-matched analysis was calculated. In the current study, there was no statistical difference in the cecal intubation time, total examination time, and insertion depth between ultrathin and conventional SBE, suggesting that insertion performance of the ultrathin SBE is equivalent to that of conventional SBE on CD.

Regarding the insertion depth, the mean insertion depth of ultrathin SBE tended to be deeper than that of conventional SBE $(p=0.09)$. This trend might be caused by passing through severe stenosis using the ultrathin SBE. Since the diameter of the ultrathin sliding tube was almost the same as the conventional SBE scope diameter (Table 3), stenosis-which is difficult to pass through with the ultrathin sliding tube-is also difficult to pass through with the conventional SBE scope. Indeed, of $20 \mathrm{CD}$ patients using the ultrathin SBE, the ultrathin sliding tube could 
not pass through severe stenosis, though the ultrathin scope could pass through in four patients. The ultrathin scope could not only pass through the stenosis, but could also be inserted approximately $30 \mathrm{~cm}$ proximally from the stenosis site; this suggests that ultrathin SBE is appropriate for use in CD patients who are expected to have severe stenosis.

In the current study, the dose of sedatives used during ultrathin SBE was significantly smaller than that in conventional SBE. No patients were taking sleeping pills at the time of SBE procedure. We could not directly assess the patient's pain and discomfort using a pain scale or visual analog scale. However, additional sedatives were administered when the patient complained of pain and discomfort during the procedure. Therefore, from these results, ultrathin SBE may reduce patients pain and discomfort more effectively than conventional SBE. The insertability and low invasiveness of the ultrathin SBE allow disease activity monitoring in $\mathrm{CD}$ patients with high patient acceptance.

This study has several limitations. First, as a retrospective single-center study with a small sample size, unintended selection bias could not be completely excluded. Second, although background factors might influence insertion of the endoscope, such as age, sex, surgical history, disease location, behavior, Harvey-Bradshaw index, and duration of disease, these were corrected with propensity score matching; however, the severity of CD was not made uniform among the included patients. Disease severity might have influenced the examination time and dose of sedatives. Third, five expert endoscopists performed enteroscopy in this study, creating the possibility of operatorassociated heterogeneity in the analysis.

In conclusion, using novel ultrathin SBE in CD patients may reduce the dose of sedatives required, and furthermore, scope insertability was equivalent to that of conventional SBE. Novel ultrathin SBE may be a less-invasive monitoring modality for the evaluation of mucosal inflammation in patients with $\mathrm{CD}$, even in cases with postoperative adhesions and inflammationinduced stenosis.

\section{CONFLICTS OF INTEREST}

The prototype single-balloon enteroscope was provided by Olympus (Tokyo, Japan). No other potential conflict of interest relevant to this article was reported.

\section{AUTHOR CONTRIBUTIONS}

Conceptualization: K.T., N.H., M.K., Y.H., R.M., K.N., K.F., Y.M., S.M., T.S., M.M., M.N. Data curation: K.T., N.H., M.K. Formal analysis: K.T., M.K. Methodology: K.T., N.H. Project administration: H.O. Visualization: K.T., N.H. Writing - original draft: K.T., N.H. Writing - review \& editing: K.T., N.H., N.Y., H.O., T.K.

\section{ORCID}

Kaoru Takabayashi

Naoki Hosoe

Yukie Hayashi

Ryoichi Miyanaga

Kosaku Nanki

Kayoko Fukuhara

Yohei Mikami

Shinta Mizuno

Tomohisa Sujino

Makoto Mutaguchi

Makoto Naganuma

Naohisa Yahagi

Haruhiko Ogata

Takanori Kanai
Motohiko Kato https://orcid.org/0000-0002-4891-8246 https://orcid.org/0000-0002-4516-5648 https://orcid.org/0000-0002-7579-1316 https://orcid.org/0000-0002-3777-2261 https://orcid.org/0000-0001-9605-2195 https://orcid.org/0000-0003-4232-0523 https://orcid.org/0000-0002-0428-8502 https://orcid.org/0000-0001-5104-4415 https://orcid.org/0000-0002-5472-2282 https://orcid.org/0000-0003-0699-6577 https://orcid.org/0000-0001-5144-6836 https://orcid.org/0000-0002-4244-3243 https://orcid.org/0000-0002-9597-4750 https://orcid.org/0000-0002-3304-3635 https://orcid.org/0000-0002-1466-4532

\section{REFERENCES}

1. Oostenbrug LE, van Dullemen HM, te Meerman GJ, Jansen PL, Kleibeuker JH. Clinical outcome of Crohn's disease according to the Vienna classification: disease location is a useful predictor of disease course. Eur J Gastroenterol Hepatol 2006;18:255-261.

2. Lescut D, Vanco D, Bonnière $P$, et al. Perioperative endoscopy of the whole small bowel in Crohn's disease. Gut 1993;34:647-649.

3. Otterson MF, Lundeen SJ, Spinelli KS, et al. Radiographic underestimation of small bowel stricturing Crohn's disease: a comparison with surgical findings. Surgery 2004;136:854-860.

4. Meucci G, Fasoli R, Saibeni S, et al. Prognostic significance of endoscopic remission in patients with active ulcerative colitis treated with oral and topical mesalazine: a prospective, multicenter study. Inflamm Bowel Dis 2012;18:1006-1010.

5. Frøslie KF, Jahnsen J, Moum BA, Vatn MH; IBSEN Group. Mucosal healing in inflammatory bowel disease: results from a Norwegian population-based cohort. Gastroenterology 2007;133:412422.

6. Schnitzler F, Fidder H, Ferrante M, et al. Mucosal healing predicts long-term outcome of maintenance therapy with infliximab in Crohn's disease. Inflamm Bowel Dis 2009;15:1295-1301.

7. Baert F, Moortgat L, Van Assche G, et al. Mucosal healing predicts sustained clinical remission in patients with early-stage Crohn's disease. Gastroenterology 2010;138:463-468.

8. Peyrin-Biroulet L, Ferrante M, Magro F, et al. Results from the 2nd Scientific Workshop of the ECCO. I: Impact of mucosal healing on the course of inflammatory bowel disease. J Crohns Colitis 2011;5:477-483.

9. Van Assche G, Dignass A, Bokemeyer B, et al. Second European evidence-based consensus on the diagnosis and management of ulcerative colitis part 3: special situations. J Crohns Colitis 2013;7:1-33.

10. Arulanandan A, Dulai PS, Singh S, Sandborn WJ, Kalmaz D. Sys- 
tematic review: safety of balloon assisted enteroscopy in Crohn's disease. World J Gastroenterol 2016;22:8999-9011.

11. Sato K, Ito S, Shigiyama F, et al. A prospective randomized study on the benefits of a new small-caliber colonoscope. Endoscopy 2012;44:746-753.

12. Horiuchi A, Nakayama Y. Unsedated ultrathin EGD by using a 5.2-mm-diameter videoscope: evaluation of acceptability and diagnostic accuracy. Gastrointest Endosc 2006;64:868-873.

13. Ai ZL, Lan CH, Fan LL, et al. Unsedated transnasal upper gastrointestinal endoscopy has favorable diagnostic effectiveness, cardiopulmonary safety, and patient satisfaction compared with conventional or sedated endoscopy. Surg Endosc 2012;26:35653572.

14. Takabayashi K, Hosoe N, Miyanaga R, et al. Clinical utility of novel ultrathin single-balloon enteroscopy: a feasibility study. Endoscopy 2019;51:468-471.

15. Ohtsuka K, Kashida H, Kodama K, et al. Diagnosis and treatment of small bowel diseases with a newly developed single balloon endoscope. Dig Endosc 2008;20:134-137.

16. May A, Nachbar L, Schneider M, Neumann M, Ell C. Push-andpull enteroscopy using the double-balloon technique: method of assessing depth of insertion and training of the enteroscopy technique using the Erlangen Endo-Trainer. Endoscopy 2005;37:6670.

17. Upchurch BR, Sanaka MR, Lopez AR, Vargo JJ. The clinical utility of single-balloon enteroscopy: a single-center experience of 172 procedures. Gastrointest Endosc 2010;71:1218-1223.
18. Pariente B, Cosnes J, Danese S, et al. Development of the Crohn's disease digestive damage score, the Lémann score. Inflamm Bowel Dis 2011;17:1415-1422.

19. Gionchetti P, Dignass A, Danese S, et al. 3rd European evidencebased consensus on the diagnosis and management of Crohn's Disease 2016: part 2: surgical management and special situations. J Crohns Colitis 2017;11:135-149.

20. Takenaka K, Ohtsuka K, Kitazume Y, et al. Utility of magnetic resonance enterography for small bowel endoscopic healing in patients with Crohn's disease. Am J Gastroenterol 2018;113:283294.

21. Ohtsuka K, Takenaka K, Kitazume Y, et al. Magnetic resonance enterography for the evaluation of the deep small intestine in Crohn's disease. Intest Res 2016;14:120-126.

22. Takenaka K, Ohtsuka K, Kitazume Y, et al. Comparison of magnetic resonance and balloon enteroscopic examination of the small intestine in patients with Crohn's disease. Gastroenterology 2014;147:334-342.

23. Atar M, Kadayifci A. Transnasal endoscopy: technical considerations, advantages and limitations. World J Gastrointest Endosc 2014;6:41-48.

24. Luo DJ, Hui AJ, Yan KK, et al. A randomized comparison of ultrathin and standard colonoscope in cecal intubation rate and patient tolerance. Gastrointest Endosc 2012;75:484-490.

25. ASGE Technology Committee, Rodriguez SA, Banerjee S, et al. Ultrathin endoscopes. Gastrointest Endosc 2010;71:893-898. 